\title{
Diffuse Alveolar Hemorrhage: A Rare Fatal Complication of Systemic Lupus Erythematosus (A Report of Two Cases)
}

\author{
Kenzle Denise G. Monsanto, MD and Geraldine T. Zamora, MD \\ Philippine General Hospital, University of the Philippines Manila
}

\begin{abstract}
We report two Filipino women with systemic lupus erythematosus (SLE) who developed diffuse alveolar hemorrhage $(\mathrm{DAH})$, a rare, life-threatening complication associated with a high mortality rate. DAH should be suspected in patients with SLE presenting with new pulmonary infiltrates, a decline in hemoglobin, hemoptysis, dyspnea, and persistent desaturation. The first patient is 23 years old and was diagnosed with SLE 8 years ago; initially presenting with malar rash, oral ulcers, nephritis, and positive antinuclear antibodies (ANA). She had a poorly controlled disease and was admitted for facial and bipedal edema due to lupus nephritis. She was given 1 gram of methylprednisolone intravenously (IV) for three consecutive days. She then became tachypneic producing bloody secretions, with desaturation and sudden decline in hemoglobin. She was given cyclophosphamide 1 gram IV and referred for plasmapheresis but eventually succumbed. The second patient is 56 years old with generalized body weakness. Laboratory workup showed nephritis, anemia, ANA, low C3, and high anti-dsDNA titers. Pulse methylprednisolone $1000 \mathrm{mg}$ was initiated. However, there was new-onset hemoptysis and desaturation and the patient was intubated. Bronchoscopy revealed diffuse bleeding on the right middle lobe and she eventually expired. Both patients with active SLE nephritis presented in this study died within days of DAH diagnosis. Hence, aside from early recognition to improve outcomes we should anticipate its possible occurrence in patients with high disease activity.
\end{abstract}

Key Words: systemic lupus erythematosus, diffuse alveolar hemorrhage, cyclophosphamide, nephritis, methylprednisolone

\section{INTRODUCTION}

Diffuse alveolar hemorrhage $(\mathrm{DAH})$ is a rare fatal complication in systemic lupus erythematosus (SLE) with a reported incidence of 0.6 to $5.4 \%$ and a high mortality rate of $70-90 \%{ }^{1,2}$ It is imperative to recognize early manifestations of DAH in patients with SLE to improve outcomes and anticipate its occurrence in patients with active disease. Its pathogenesis is poorly understood but is presumed to be directed at immune complex deposition in alveolar walls., Risk factors for the development of DAH include an active disease with Systemic Lupus Erythematosus Disease Activity Index (SLEDAI) score above 10, hypoalbuminemia, low complement levels, high anti-dsDNA titers, and concomitant lupus nephritis. DAH manifests as hemoptysis, new drop in hemoglobin, hypoxemic respiratory failure, and diffuse pulmonary infiltrates on radiography. DAH presents a diagnostic and therapeutic dilemma due to the variety of disorders that may be included in the differential diagnoses. ${ }^{5}$

Chest radiograph may reveal focal or diffuse alveolar

Corresponding author: Kenzle Denise G. Monsanto, MD Philippine General Hospital University of the Philippines Manila

Taft Avenue, Ermita, Manila 1000, Philippines

Email:kgmonsanto@up.edu.ph infiltrates; however, these may be non-specific. A chest computed tomography scan (CT-scan) may be needed to confirm and illustrate the extent of the disease. Bronchoalveolar lavage fluid (BALF) is usually hemorrhagic; however, 
hemosiderin-laden macrophages may accumulate later, usually after $2-4$ days of DAH diagnosis.,3

High-dose corticosteroids and immunosuppressive agents are the cornerstones of therapy, but there are no reported guidelines for DAH. Its management remains to be individualized. Cyclophosphamide use remains controversial as some studies reported increased survival rate, as well as increased mortality rate through the development of primary respiratory tract infection. ${ }^{4-6}$ Plasmapheresis is commonly used for other autoimmune-related causes of vasculitis with pulmonary bleeding but has not been proven effective for DAH associated with SLE. ${ }^{6,7}$ Rituximab, an emerging treatment, was employed in some case reports but is still without ample evidence to support its use; hence, management is individualized. ${ }^{8,9}$ The prognosis of this disease is not completely defined because reports differ in the clinical characteristics and outcomes of the disease. In addition, these patients are usually excluded from large trials. ${ }^{10}$

\section{CASE 1}

A 23-year-old Filipino woman who has been diagnosed with SLE for 8 years developed facial puffiness two months before the consult. She later presented with bipedal edema, easy fatigability, and difficulty in ambulation; hence, she was prescribed prednisone $50 \mathrm{mg}$ per day, mycophenolate mofetil 2 grams per day, furosemide $10 \mathrm{mg}$ daily. She had no history of fever, rash, or joint pains. However, there were body malaise and vomiting necessitating hospital admission.

On admission, she had stable vital signs, oral ulcers, and anasarca. Laboratory tests revealed normocytic normochromic anemia with hemoglobin ( $\mathrm{Hgb}, 96 \mathrm{~g} / \mathrm{L})$, normal white cell count (WBC, 10x10\%/L), normal platelet count, Coomb's test, and elevated erythrocyte sedimentation rate (ESR, $91 \mathrm{~mm} / \mathrm{hr}$ ). There was azotemia (BUN $26.7 \mathrm{mmol} / \mathrm{L}$; creatinine $787 \mathrm{umol} / \mathrm{L})$ and hypoalbuminemia $(2 \mathrm{~g} / \mathrm{dl})$. She had nephrotic-range proteinuria $(8 \mathrm{~g})$, low $\mathrm{C} 3(0.698 \mathrm{~g} / \mathrm{L})$ and elevated anti-dsDNA (130 IU/ml). The chest radiograph (Figure 1) was normal, procalcitonin was low, and nasopharyngeal swab for severe acute respiratory syndrome coronavirus 2 (SARS-CoV-2) RT-PCR was negative. The impression at this time was active lupus nephritis; hence, pulsed methylprednisolone 1 gram IV was started on the $1^{\text {st }}$ hospital day (HD) for 3 consecutive days. However, on the $4^{\text {th }}$ hospital day, the patient had sudden onset of dyspnea with persistent desaturations around 50-60\% despite oxygen support, with new-onset crackles on all lung fields, and with serous bullae on the right foot. She was intubated and mechanically ventilated, but still with persistent desaturations and bloody secretions per endotracheal tube. $\mathrm{CBC}$ revealed a $33 \mathrm{~g} / \mathrm{L}$ drop of $\mathrm{Hgb}$ at $65 \mathrm{~g} / \mathrm{L}$, hematocrit (Hct) at $20 \%$, with normal $\mathrm{WBC}$ and platelet count. Arterial blood gas (ABG) level revealed uncompensated metabolic acidosis with severe hypoxemia. A chest radiograph (Figure 2) revealed bilateral infiltrates with bilateral pulmonary

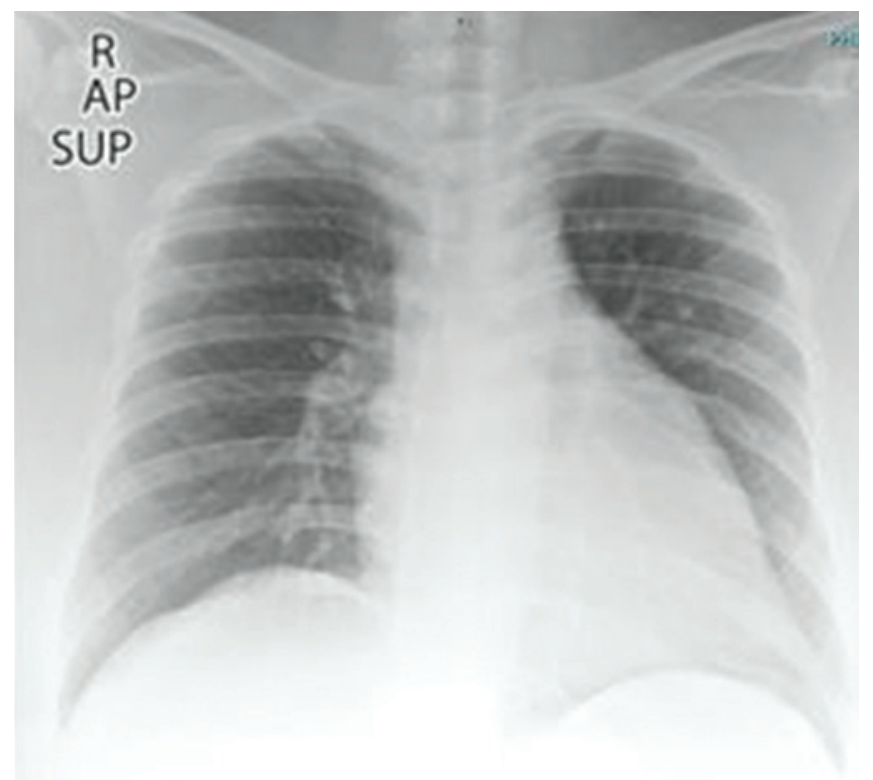

Figure 1. Chest X-ray (admission): cardiomegaly, no infiltrates.

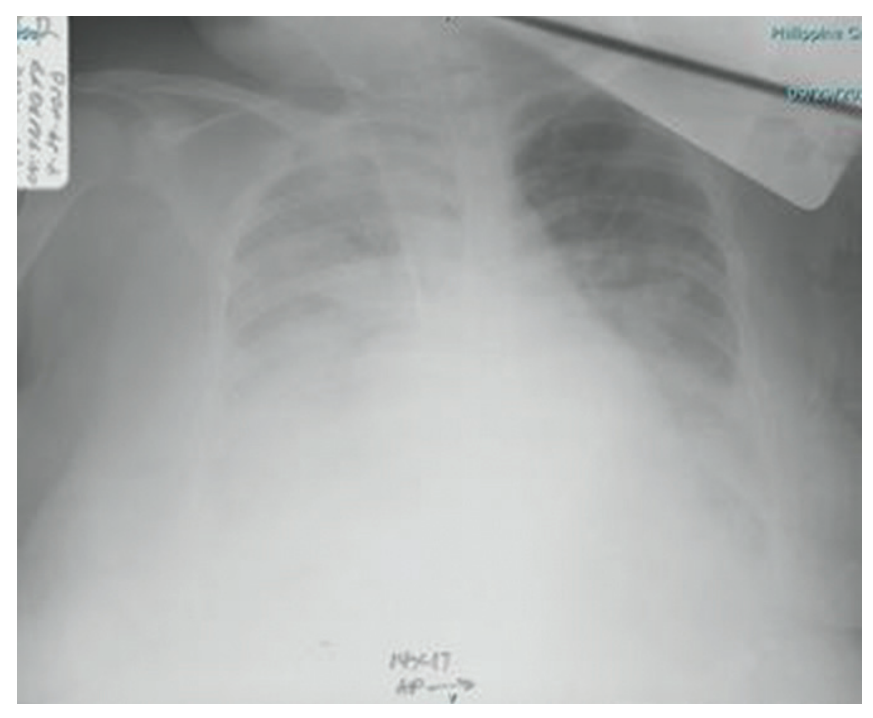

Figure 2. Chest $\mathrm{X}$-ray $4^{\text {th }} \mathrm{HD}$ : bilateral infiltrates with pulmonary congestion.

congestion. Repeat SARS-CoV-2 RT-PCR was negative. Electrocardiogram and 2D echocardiography were normal (ejection fraction 60\%). A high-resolution CT scan (HRCT) revealed no evidence of pulmonary embolism, with ground glass densities, consolidation with diffuse interlobular septal thickening compatible with DAH. Cultures were obtained and empiric broad-spectrum antibiotics were initiated. The patient was also given sodium bicarbonate drip, packed RBC transfusion, hemodialysis, and intravenous cyclophosphamide. The patient eventually succumbed on the $10^{\text {th }}$ hospital day. 


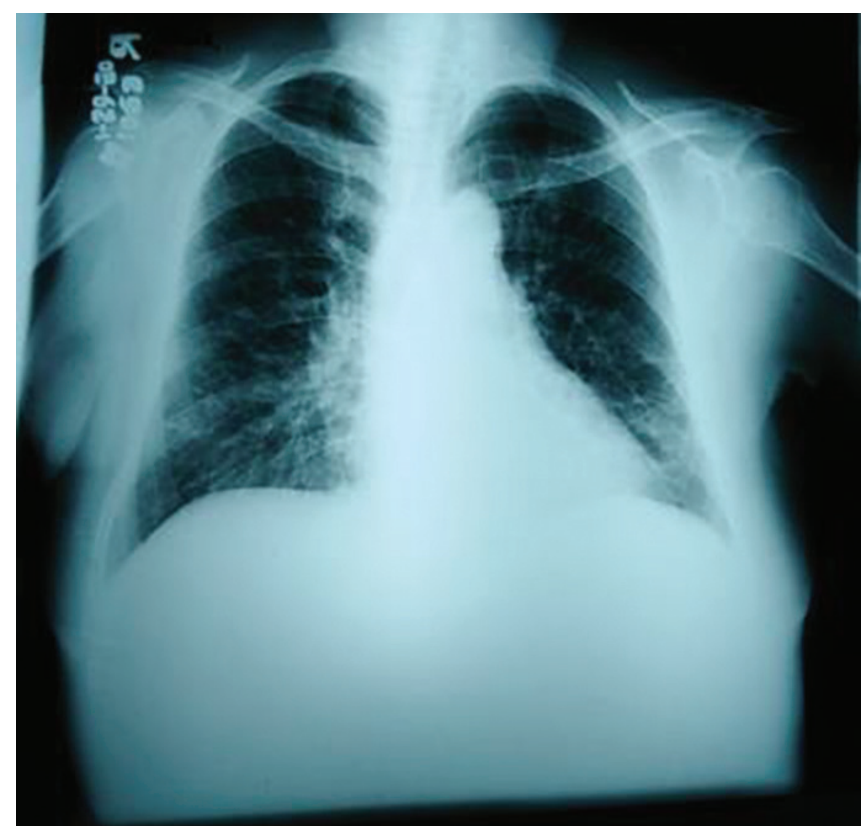

Figure 3. Chest X-ray (admission): Prominent pulmonary interstitial markings.

\section{CASE 2}

A 56-year-old Filipino woman presented with generalized weakness and easy fatigability for two months. Two weeks before admission (PTA), she had increased generalized weakness associated with pallor. $\mathrm{CBC}-$ showed normocytic normochromic anemia with a hemoglobin of $84 \mathrm{~g} / \mathrm{L}$ for which ferrous sulfate supplementation was prescribed. Eight days PTA, the symptoms persisted with loss of appetite; hence, she sought admission at a local hospital. Due to a hemoglobin level of $71 \mathrm{~g} / \mathrm{L}$, she was advised transfusion but there was difficulty in cross-matching; hence, she was transferred to a tertiary institution. The patient had no known comorbidities and no other associated symptoms such as rash, alopecia, fever, cough, dyspnea, chest pain, abdominal pain, joint pains, or bipedal edema.

At the emergency department, the patient was afebrile with stable vital signs and no overt signs of bleeding. She had symmetric bilateral clubbing, but no cyanosis or desaturations were noted. Her lungs were clear (Figure 3). She was managed as symptomatic anemia and packed RBC transfusion was ordered. Laboratory results showed hemolytic anemia with $\mathrm{Hgb}$ of $56 \mathrm{~g} / \mathrm{L}$, Hct of $16 \%$ with normal platelet count, positive ANA IF (1:160), positive rheumatoid factor, elevated C-reactive protein $(\mathrm{CRP})>12 \mathrm{mg} / \mathrm{dl}$, hypoalbuminemia $(2.4 \mathrm{~g} / \mathrm{dl})$, low $\mathrm{C} 3(0.2 \mathrm{~g} / \mathrm{L})$ and elevated anti-dsDNA (229 IU/ml). Urinalysis showed trace proteinuria and 24-hour total urinary protein was $600 \mathrm{mg} /$ day. The antiphospholipid antibodies panel, hepatitis profile, and thyroid function tests were unremarkable. The working impression was SLE with nephritis and hydrocortisone 100

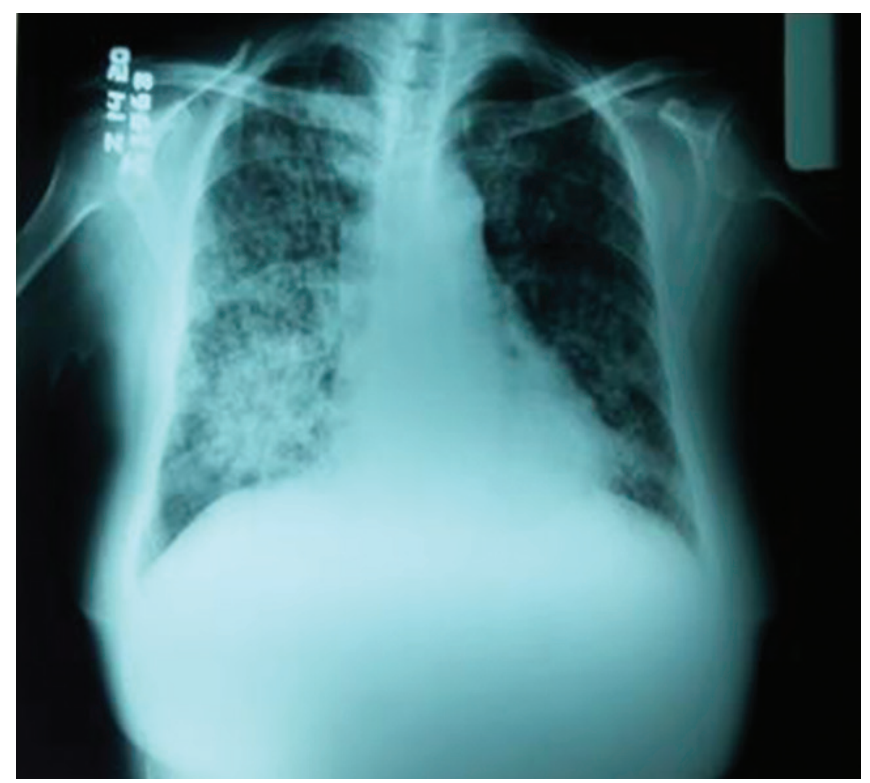

Figure 4. Chest X-ray $14^{\text {th }}$ HD: Patchy reticulonodular infiltrates at bilateral lung fields, bilateral with relative sparing of bilateral apices and base.

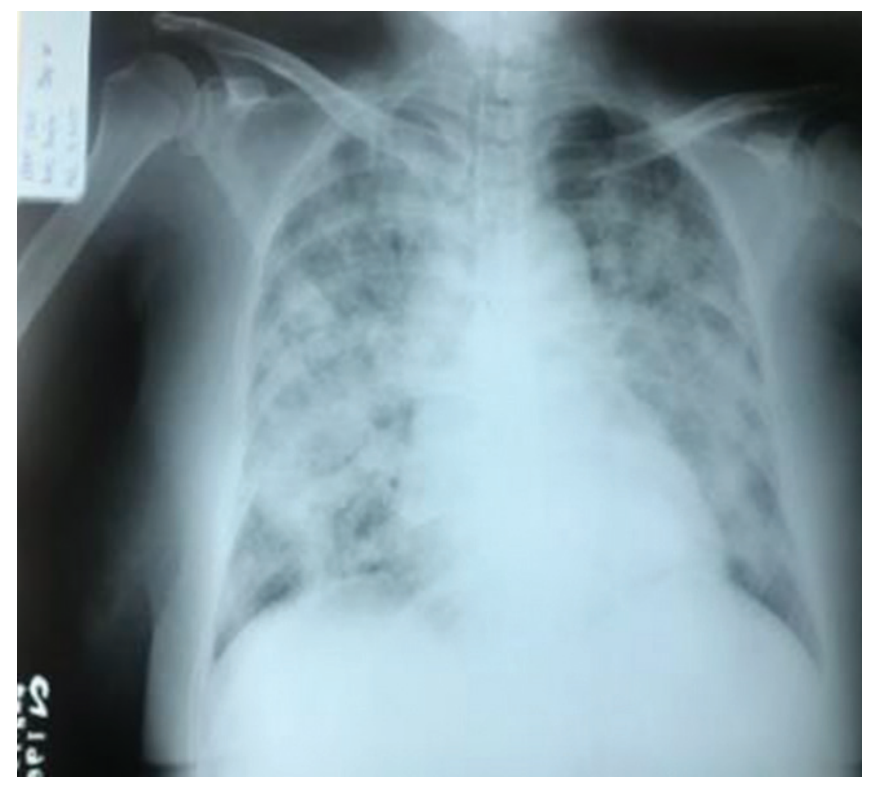

Figure 5. Chest X-ray $15^{\text {th }} \mathrm{HD}$ : Bilateral patchy reticulonodular infiltrates.

$\mathrm{mg}$ IV every 8 hours $(1 \mathrm{mg} / \mathrm{kg} /$ day prednisone equivalent) was started. Bone marrow samples submitted for cytogenic studies and red cell antibody determination showed normal results. The patient was still anemic with a hemoglobin of $64 \mathrm{~g} / \mathrm{L}$ despite high dose corticosteroids; hence, methylprednisolone pulse therapy 1 gram IV for 3 days was initiated.

On the $14^{\text {th }} \mathrm{HD}$, the patient had new-onset hemoptysis, pallor, BP elevation at $140 / 90 \mathrm{mmHg}$, tachypnea (40 cpm), tachycardia (113 bpm), with no fever. She had oxygen 
desaturation as low as $88-89 \%$, with bilateral coarse crackles; hence, she was intubated and hooked to a mechanical ventilator. There were bloody secretions seen per endotracheal tube. A chest radiograph showed new patchy, reticulonodular infiltrates in the right lower lung field (Figure 4). Broadspectrum antibiotics were empirically started. An emergency CT pulmonary angiogram was requested; however, the patient could not tolerate transport to the radiology department. On the $15^{\text {th }} \mathrm{HD}$, a repeat chest radiograph (Figure 5) revealed bilateral patchy reticulonodular infiltrates and bronchoscopy was done that showed diffuse bleeding of bilateral lung fields, most prominent in the right middle lobe. Bronchial washing specimens revealed PMN 0-2 with gram-positive diplococci and cytology, negative for AFB. Blood culture was negative. Samples were sent for culture and TB PCR. Plasmapheresis was offered as the next therapeutic option for the patient, however, on the $16^{\text {th }}$ hospital day, the patient was bradycardic with unappreciated BP. ACLS was done but the patient eventually expired.

\section{DISCUSSION}

Diffuse alveolar hemorrhage is a diagnostic and therapeutic dilemma in SLE that can quickly be fatal. We presented two patients with SLE with active nephritis who developed DAH and eventually died in a matter of days. The incidence of DAH varies based on reported data on various case series, ranging from $0.63 \%$ to $5.4 \%$. In a case-control study in 2020 by Sun Yidou et.al with 4744 patients with SLE, DAH prevalence was $2 \%$, with a young female predominance of $87.2 \%$ and the median age at DAH diagnosis of 29 years, like our first patient who falls within the same age range. DAH manifested as the initial presentation in $18.8 \%$ of cases and happened within the first year of SLE diagnosis in $59.6 \%$ of cases. ${ }^{6}$ The most common presentations were dyspnea, hypoxemia, and hemoptysis, all of which were seen in our patient. Lupus nephritis, which was the predominant disease activity in both patients; hematological involvement (such as hemolytic anemia in the first patient); and elevated c-reactive protein (CRP) were described as independent risk factors of DAH. Both patients exhibited elevated acute phase reactants and acute hemoglobin decline of at least 2-3 $\mathrm{g} / \mathrm{dl}$. While thrombocytopenia and leukopenia are strongly associated with $\mathrm{DAH}, 2,8$ these findings were not present in both of our patients. In related studies by Quintana et al., Ming-Yang et al., and Aldhoubi et al., majority of patients with SLE with DAH exhibited low levels of C3, high anti-dsDNA titer, and negative for ANCA, ${ }^{1,2,11}$ which were similar to our patient's laboratory results.

Imaging tests are essential in establishing a diagnosis. Chest radiography usually shows bilateral airspace opacities. If initial imaging is ambiguous but high clinical suspicion for DAH is apparent, a CT scan should be done, as in the first patient whose CXR was normal. Common CT findings include diffuse and patchy ground-glass densities along with diffuse nodular opacities. ${ }^{12}$ The clinical features described in the literature were also apparent in our patients. A high-resolution CT scan (HRCT) revealed ground-glass densities and consolidation with diffuse interlobular septal thickening compatible with DAH.

Infections can be associated with DAH and should always be included in the investigation. Patients with DAH and concurrent infections generally show a poor prognosis. ${ }^{5,13}$ The clinical signs may be similar to heart failure, infections, or acute lupus pneumonitis. In a study by Rojas, et al. that evaluated the infection rate in patients diagnosed with DAH, they demonstrated that $57 \%$ of cases had infections including Pseudomonas aeruginosa, Serratia marcescens, Citrobacter freundii, and Aspergillus fumigatus. These findings support the early initiation of broad-spectrum antibiotics and continuous surveillance for possible emerging infections. Our first patient was worked up for COVID-19 infection but which turned out to be negative. Both patients were treated empirically with broad-spectrum antibiotics while awaiting blood and sputum/tracheal cultures. Both were also worked up for heart failure. Bronchoscopy was performed in most patients with DAH and revealed hemorrhage in $66 \%$ of patients, hemosiderin-laden macrophages in $15 \%$, and $7 \%$ had a positive result for infection specifically Pneumocystis, cytomegalovirus, and Legionella baumanii. ${ }^{14}$ This is consistent with our second patient who underwent bronchoscopy that showed diffuse bleeding on both lungs, most prominently in the right middle lobe.

There is a scarcity of evidence in clinical trials to treat patients with SLE-associated DAH and management remains individualized in some case studies. The most frequently implemented therapeutic management are methylprednisolone, cyclophosphamide, and plasmapheresis. Empirical studies have indicated the survival rate is higher for patients receiving a dose of methylprednisolone above what is conventionally used (4-8 g instead of $3 \mathrm{~g}$ ) and concomitant use of cyclophosphamide has shown to improve survival. ${ }^{8,13}$ Our first patient was given pulse methylprednisolone therapy $(3 \mathrm{~g})$ while the second patient was on high-dose steroid treatment $(1 \mathrm{mg} / \mathrm{kg} /$ day $)$ in the early course of admission; however, both patients expired. In another study, a high mortality rate was apparent in patients receiving immunosuppressive treatment while on a mechanical ventilator. This may be attributed to the severity of the case on presentation. ${ }^{5}$ In comparison, both our patients were intubated and attached to a mechanical ventilator and were given immunosuppression but expired within days of DAH diagnosis.

Plasmapheresis is efficacious therapy for causes of DAH such as ANCA-associated vasculitis, anti-glomerular basement disease, and antiphospholipid syndrome. Plasmapheresis may be offered to patients who are refractory to pulse steroids and intravenous cyclophosphamide therapy. ${ }^{7,13}$ The patients presented in this series were given relatively the same treatment modalities but still had rapid deterioration. 
The first patient was offered plasmapheresis; however, it was not given since our patient eventually succumbed. Other therapeutic options were proposed which included rituximab, a monoclonal antibody against CD20-positive B lymphocytes is used in refractory lupus; however, its role as first-line therapy is not well established in randomized trials to date. ${ }^{15}$

\section{CONCLUSION}

We presented two cases of diffuse alveolar hemorrhage as a rare complication of SLE with a high rate of mortality. It is advisable to anticipate its possible occurrence inpatient with associated risk factors, including nephritis, hematologic activity, elevated CRP, and infections, as well as to recognize it early. A chest CT scan could be performed if the chest radiograph is normal or ambiguous but the probability of DAH is high. Prompt targeted management of the underlying autoimmune process and various other concomitant complications to improve outcome and quality of life is imperative.

\section{Statement of Authorship}

Both authors contributed in the conceptualization of work, acquisition and analysis of data, drafting and revising and approved the final version submitted.

\section{Author Disclosure}

Both authors declared no conflicts of interest.

\section{Funding Source}

The study has no funding support.

\section{REFERENCES}

1. Quintana JH, Aragón CC, Santos VA, de Las Salas A, Tafúr RA, Aguirre-Valencia D, Cañas CA, Tobón GJ. Diffuse alveolar hemorrhage: a cohort of patients with systemic lupus erythematosus. J Clin Rheumatol. 2020 Oct; 26(7S Suppl 2):S153-S157. doi: 10.1097/ RHU.0000000000001228. PMID: 31895107.

2. Al-Adhoubi NK, Bystrom J. Systemic lupus erythematosus and diffuse alveolar hemorrhage, etiology and novel treatment strategies. Lupus. 2020 Apr; 29(4):355-63. doi: 10.1177/0961203320903798. PMID: 32036761; PMCID: PMC7436451.

3. Newsome BR, Morales JE. Diffuse alveolar hemorrhage. South Med J. 2011 Apr; 104(4):269-74. doi: 10.1097/SMJ.0b013e3182126d3b. PMID: 21606695.
4. Zamora MR, Warner ML, Tuder R, Schwarz MI. Diffuse alveolar hemorrhage and systemic lupus erythematosus. Clinical presentation, histology, survival, and outcome. Medicine (Baltimore). 1997 May; 76(3):192-202. doi: 10.1097/00005792-199705000-00005. PMID: 9193454.

5. Lara AR, Schwarz MI. Diffuse alveolar hemorrhage. Chest. 2010 May; 137(5):1164-71. doi: 10.1378/chest.08-2084. PMID: 20442117.

6. Sun Y, Zhou C, Zhao J, Wang Q, Xu D, Zhang S, et al. Systemic lupus erythematosus-associated diffuse alveolar hemorrhage: a singlecenter, matched case-control study in China. Lupus. 2020 Jun; 29(7): 795-803. doi: 10.1177/0961203320920715. PMID: 32321345.

7. de Holanda BA, Barreto IG, de Araujo IS, de Araujo DB. Alveolar hemorrhage as the initial presentation of systemic lupus erythematosus. Reumatologia. 2016; 54(5):264-6. doi: 10.5114/reum.2016.63668. PMID: 27994272; PMCID: PMC5149575..

8. Kazzaz NM, Coit P, Lewis EE, McCune WJ, Sawalha AH, Knight JS. Systemic lupus erythematosus complicated by diffuse alveolar haemorrhage: risk factors, therapy and survival. Lupus Sci Med. 2015 Sep 23; 2(1):e000117. doi: 10.1136/lupus-2015-000117. PMID: 26430514; PMCID: PMC4586940.

9. Wang CR, Liu MF, Weng CT, Lin WC, Li WT, Tsai HW. Systemic lupus erythematosus-associated diffuse alveolar haemorrhage: a singlecentre experience in Han Chinese patients. Scand J Rheumatol. 2018 Sep; 47(5):392-9. doi: 10.1080/03009742.2017.1420817. PMID: 29916287.

10. Mirouse A, Parrot A, Audigier V, Demoule A, Mayaux J, Geri G, et al. Severe diffuse alveolar hemorrhage related to autoimmune disease: a multicenter study. Crit Care. 2020; 24:231. https://doi.org/10.1186/ s13054-020-02936-0

11. Chang MY, Fang JT, Chen YC, Huang CC. Diffuse alveolar hemorrhage in systemic lupus erythematosus: a single center retrospective study in Taiwan. Ren Fail. 2002 Nov; 24(6):791-802. doi: 10.1081/jdi-120015681. PMID: 12472201.

12. Cheah FK, Sheppard MN, Hansell DM. Computed tomography of diffuse pulmonary haemorrhage with pathological correlation. Clin Radiol. 1993 Aug; 48(2):89-93. doi: 10.1016/s0009-9260(05)81078-5. PMID: 8004902.

13. Martinez-Martinez MU, Sturbaum AK, Alcocer-Varela J, MerayoChalico J, Gómez-Martin D, Gómez-Bañuelos Jde J, et.al. Factors associated with mortality and infections in patients with systemic lupus erythematosus with diffuse alveolar hemorrhage. J Rheumatol. 2014 Aug; 41(8):1656-61. doi: 10.3899/jrheum.130927. Epub 2014 Jul 1. PMID: 24986849.

14. Ednalino C, Yip J, Carsons SE. Systematic Review of Diffuse Alveolar Hemorrhage in Systemic Lupus Erythematosus: Focus on Outcome and Therapy. J Clin Rheumatol. 2015 Sep; 21(6):305-10. doi: 10.1097/ RHU.0000000000000291. PMID: 26308350.

15. Dhungana A, Shrestha P, Shrestha BD, Baral A, Sayami G. Diffuse Alveolar Hemorrhage as Initial Presentation of Systemic Lupus Erythematosus: A Case Report. JNMA J Nepal Med Assoc. 2018 Nov-Dec; 56(214):970-3. PMID: 31065146.;569214:970 